\title{
Nonlinear Production, ABATEMENT, POLLUTION AND MATERIALS BALANCE RECONSIDERED
}

\author{
RUEDIGER PETHIG
}

CESIFO WORKING PAPER NO. 1549

CATEGORY 8: RESOURCES AND ENVIRONMENT

SEPTEMBER 2005

An electronic version of the paper may be downloaded

- from the SSRN website:

www.SSRN.com

- from the CESifo website: www.CESifo-group.de 


\title{
NONLINEAR PRoduction, ABATEMENT, POLLUTION AND MATERIALS BALANCE RECONSIDERED
}

\begin{abstract}
In the environmental economics literature the standard approach of modeling nonlinear production and abatement processes is to treat waste emissions "simply as another factor of production" (Cropper and Oates 1992). That approach doesn't map the materials flow involved completely and hides, moreover, the exact links between production, residuals generation and abatement. This paper shows that production functions with emissions treated as inputs can be reconstructed as a subsystem of a comprehensive production-cum-abatement technology that is in line with the materials-balance principle. In a simple economy with full regard of the materials flow it also explores the consequences for allocative efficiency and efficiency-restoring taxation of multiple and interdependent residuals generated in the transformation processes of production, abatement and consumption. Finally, the paper demonstrates that efficiency may require setting the emissions tax rate above or below conventionally defined marginal abatement cost if the residual subject to abatement is not the only residual causing pollution.
\end{abstract}

JEL Code: Q50, Q52.

Keywords: residuals, abatement, pollution, materials balance.

Ruediger Pethig

University of Siegen

Department of Economics

FB5

Hölderlinstr. 3

57068 Siegen

Germany

Pethig@vwl.wiwi.uni-siegen.de 


\section{Nonlinear production, abatement, pollution and materials balance reconsidered ${ }^{1}$}

Rüdiger Pethig, University of Siegen

\section{The problem}

As is well-known from the law of mass conservation, the flow of materials taken from the environment for economic uses generates a flow of materials from the economy back into the environment that is of equal weight (after accounting for time delays). The economic activities of production and consumption are merely processes of transforming materials that only change the physical and chemical attributes and the composition of the materials flow. Quite obviously, the composition of the flow of residuals from the economy into the environment is of great significance because different kinds of residuals differ in their detrimental impact on the environment. $^{2}$

As a consequence, a sensible strategy for alleviating the problem of environmental degradation is to control the process of materials transformation by reducing the emission of the most harmful pollutants through residuals abatement activities. Like production and consumption, this activity is a process of transforming materials subject to the materials-balance principle: the weight of all material outputs of that process equals the weight of all material inputs.

In their seminal paper on 'production, consumption and externalities', Ayres and Kneese (1969) made a strong case for the need of a consistent and encompassing application of the materials-balance principle to all transformation processes. In their formalized materialsbalance approach they employed linear technologies with fixed input-output coefficients but since then the profession has revealed a preference for modeling non-linear rather than linear technologies. In fact, the notion and empirical evidence of strictly increasing (real) marginal abatement costs is at the core of many pollution control studies.

To be sure, it is possible to bring non-linear (abatement) technologies into line with materialsbalance requirements, too. This has been demonstrated in various previous studies the most general and ambitious of which probably is Krysiak and Krysiak (2003). Yet fully regarding the materials-balance principle in theoretical analysis comes at the cost of enormous addi-

\footnotetext{
${ }^{1}$ Helpful comments by Thomas Eichner, Reyer Gerlagh and by two anonymous referees are gratefully acknowledged. Remaining errors are the author's sole responsibility.

2 A more detailed analysis would need to focus on further determinants such as the medium of discharge and the local environmental medium's assimilative capacity.
} 
tional complexity which tends to prevent the derivation of informative results. To avoid such complexities many environmental economists became reluctant to explicitly and properly regard the materials-balance principle as the correct theoretical foundation of their analyses (Pezzey and Toman 2002, 202; Pethig 2003). Therefore Ayres and Kneese's (1969, 283) verdict still applies to much of the present work that production processes are viewed "... in a manner that is somewhat at variance with the law of conservation of mass".

To be more specific, consider the simple production function $Y: \mathbb{R}_{+}^{3} \rightarrow \mathbb{R}_{+}$with

$$
y=Y(e, \ell, m)
$$

where two inputs, labor $\ell$ and material $m$, are employed to produce two outputs as joint products, a wanted consumer good, $y$, and an unwanted production residual, $e$ (with $e$ for emissions). This type of technology was already applied in the early 1970s, e. g. by Forster (1972) and Klevorick and Cramer (1972). Varying grossly in its degree of generality, it became a widespread and accepted tool of analysis within few years (e. g. in Mäler 1974, Pethig 1975, Baumol and Oates 1975).

In their survey on environmental economics, Cropper and Oates (1992) refer to the production function (1) as the standard approach in the environmental economics literature ${ }^{3}$. They observe that the treatment of waste emissions "simply as another factor of production ... seems reasonable since attempts ... to cut back on waste discharge will involve the diversion of other inputs to abatement activities - thereby reducing the availability of these other inputs for the production of goods" (Cropper and Oates 1992, p. 678). This citation reveals these authors' awareness of technology (1) as a concept that implicitly involves both the generation of a production residual and an abatement activity. Cropper and Oates (1992, p. 678) also find it reasonable "...to assume the usual curvature properties ..." that is, they require function $Y$ from (1) to exhibit the

Properties (Y): $Y: \mathbb{R}_{+}^{3} \rightarrow \mathbb{R}_{+}$is concave and satisfies $Y_{e}>0, Y_{\ell}>0$ and $Y_{m}>0$.

It is not clear, however, what exactly is the link between the production of a consumer good, residuals generation and abatement which Cropper and Oates conjecture as being hidden in (1). To put it differently, it is not clear how an explicit and comprehensive analysis of interde-

\footnotetext{
3 As compared to our equation (1), the equation (2) in Cropper and Oates (1992, p. 678) is slightly more general in that they allow for an arbitrarily large vector of conventional inputs (which is reduced to the two-dimensional vector $(\ell, m)$ in (1)) and allow the level of pollution to be a negative productivity-reducing externality. This externality is omitted in the present paper to keep the analysis simple.
} 
pendent production, residuals generation and abatement would relate to production functions of type (1). ${ }^{4}$

The main objectives of the present paper are (i) to undertake a fresh exploration of that issue with the important qualification to keep the analysis strictly (and explicitly!) in line with the materials-balance principle and (ii) to assess the consequences of (i) for allocative efficiency and pollution control. An immediate implication of adopting a rigorous materials-balance perspective is to insist that the treatment of emissions in (1) as 'conventional inputs in production functions' is only acceptable as a convenient though purely formal analytical device not meant to deny the emissions' true nature as unwanted by-products generated in the process of producing wanted goods and then discharged into the environment.

To further clarify this point suppose $\ell$ and $m$ are constant in (1). Since $Y_{e}$, the marginal abatement cost in terms of the wanted output, is positive, one can choose from a menu of good things, $y$, and bad things, $e$, but more of the good inadvertently comes along with more of the bad. While this property of (1) serves the needs of model building in environmental economics well, (1) is less appealing, if not outright embarrassing, regarding its materialsbalance implications. To see that suppose all units of the outputs $y$ and $e$ as well as all units of input $m$ are defined such that each unit is equal to one unit of weight. Suppose further that the technology (1) is understood as a process of transforming the material $m$ into the outputs $y$ and $e$. With these qualifications one may wish to know whether (1) can be considered the description of a transformation process that involves no material inputs other than $m$ and no material outputs other than $y$ and $e$. The answer is an outright and definitive no. (1) would blatantly violate the materials-balance principle, since that principle requires $m=e+y$ if no other inputs and outputs are involved. An obvious implication of $m=e+y$ is $d y / d e=-1$ for constant $m$, which is, of course, inconsistent with properties (Y) that requires $Y_{e}>0$.

It is not our intention to join in the chorus of those who declare all pieces of research in environmental economics fundamentally flawed that are found guilty of not properly regarding the materials-balance principle. We rather aim at answering the intriguing question whether, and if so how, the production activity (1) can be reconciled with the materials-balance principle. We will show that (1) can be reconstructed, indeed, as part of a comprehensive productioncum-abatement technology that is in line with the materials-balance principle.

\footnotetext{
${ }^{4}$ The only theoretical inquiry into that issue we are aware of is offered in Siebert et al. (1980). Yet these authors fail to fully account for the materials-balance principle which is why their approach is of limited relevance for our subsequent analysis.
} 
Maintaining the standard technological assumptions of concavity, non-linearity, ${ }^{5}$ and smooth factor substitution, the proper regard of materials-balance requirements will make it necessary to also account for residuals other than the production residuals represented by the variable $e$ in (1). We will also demonstrate that these additional interdependent residuals do not render incorrect the conventional analysis of pollution control based on (1) if and only if their emission doesn't contribute to environmental degradation. Insofar we provide a rigorous rationale and justification for conventional model building. However, if the emitted production residuals, $e$, are not the only pollutants, the conditions determining allocative efficiency will be shown to differ markedly from those derived in conventional analysis. In that case, the conventional marginal cost of abating production residuals, $Y_{e}$, will turn out to deviate from the social marginal cost of abating these residuals because we deal with a pollution problem involving multiple and interdependent pollutants. This finding will be shown to have non-trivial implications for efficiency-restoring tax schemes.

Section 2 introduces a comprehensive technology of production and residuals abatement based on the materials-balance principle, and we will rigorously derive the production function (1) as a proper though incomplete technological subsystem of the comprehensive production-cum-abatement technology. Moreover, the entire comprehensive production-cumabatement technology will be shown to be completely represented by (1) and two further production functions mapping the domain of (1) into the abatement residuals. In Section 3 we will incorporate the comprehensive production-cum-abatement technology developed in Section 2 into a simple economy subject to pollution, and we will derive the pertaining rules for an efficient allocation. If residuals other than (unabated) production residuals also cause pollution, the optimality rules become complex, since all these pollutants are generated in strict technological interdependence. Section 4 explores the consequences of that interdependence for the design of efficiency-restoring tax schemes. Taking the conventional Pigouvian tax rule as a benchmark we show that if residuals other than production residuals contribute to pollution in addition to the latter, it is not efficient, in general, to set the tax on production residuals equal to the conventional marginal abatement cost, $Y_{e}$.

\footnotetext{
5 Ayres and Kneese (1969) developed their 'materials balance approach' in a model with strictly linear production processes. That makes it quite easy to keep track of material balance but fails to account for realistic substitution and transformation possibilities. See also Pethig (2003).
} 


\section{Abatement and production in line with materials balance}

Suppose during the process of producing a consumer good a production residual is generated with each unit equal to one unit of weight. This residual is assumed to be an unwanted product, useless for consumptive and productive purposes, and harmful if discharged into the environment. It is therefore a candidate for abatement. Although it is quite common to equate abatement with disappearance into the void, abatement is clearly an activity of combining scarce inputs, both material inputs and services, for the purpose of transforming the residuals under consideration into outputs with different physical and/or chemical attributes. Abatement does "not destroy the residuals but only alters their form" (Ayres and Kneese 1969, 283).

To formalize such an abatement activity in a very simple way, let $a$ be the amount of the production residuals to be abated. Technically speaking, $a$ is an input in the transformation process to be described and needs to be combined with other inputs to make the transformation work. Suppose two other inputs are necessary: a service, called labor, $\ell_{a}$, and a physical input, called material, $m_{a}$. With these 'ingredients', the aim of abatement is to use labor for transforming both material inputs, $a$ and $m_{a}$, into two distinct kinds of material residuals denoted abatement residuals, which are assumed to differ from production residuals, $a$, and material, $m_{a}$, in some significant way. But it is also obvious that with given positive amounts $\ell_{a}$ and $m_{a}$ one cannot transform arbitrarily large amounts of production residuals into abatement residuals. In fact, we assume that there is a technology $A:\left(\ell_{a}, m_{a}\right) \rightarrow a$ such that the amount $A\left(\ell_{a}, m_{a}\right)$ of the production residual can be abated when labor input is $\ell_{a}$ and material input is $m_{a}$. We assume the function $A$ to have the

Properties (A): $A: \mathbb{R}_{+}^{2} \rightarrow \mathbb{R}_{+}$is concave and satisfies $A\left(0, m_{a}\right)=A\left(\ell_{a}, 0\right)=0$, $A_{\ell}>0, A_{m}>0, A_{\ell \ell}<0, A_{m m}<0$ and $A_{\ell m}>0$.

$A_{\ell}>0$ and $A_{m}>0$ implies that $\ell_{a}$ and $m_{a}$ can be substituted against each other when a given amount of production residuals is to be abated. To sum up, $\left(a, \ell_{a}, m_{a}\right)$ is considered a feasible abatement activity, if $A\left(\ell_{a}, m_{a}\right)=a$.

The next step is to specify the generation of abatement residuals. How exactly and in which amounts abatement residuals are generated is an engineering issue that will not be pursued here. For expository purposes we will consider here the very simple case where all 'abated' production residuals are turned into one kind of abatement residuals, called abatement residu- 
als of type 1, and where all material input used in the process of abatement is turned into another kind of abatement residuals, called abatement residuals of type 2. Denoting the amounts of abatement residuals by $r_{a_{1}}$ and $r_{a_{2}}$, respectively, our simple technological assignment is $a=r_{a_{1}}$ and $m_{a}=r_{a_{2}}$.

The technology of producing consumer goods is introduced in form of the production function

$$
y=F\left(\ell_{y}, m_{y}\right) \text {, }
$$

where $\ell_{y}$ denotes labor input, $m_{y}$ denotes material input and $y$ is the amount of consumer good produced.

Properties (F): $F: \mathbb{R}_{+}^{2} \rightarrow \mathbb{R}_{+}$is concave and satisfies $F\left(0, m_{y}\right)=F\left(\ell_{y}, 0\right)=0$, $\left.F_{\ell}>0, F_{m} \in\right] 0,1\left[, F_{\ell \ell}<0 \quad F_{m m}<0\right.$ and $F_{\ell m}>0 .^{6}$

The constraint $\left.F_{m} \in\right] 0,1[$ is absent from conventional production functions and therefore demands an explanation. Our simple production model assumes that there is one and only one material input whose quantity $m_{y}$ is transformed into at least two different outputs, the consumer good (quantity $y$ ) and some production residuals (specified below), since the entropy law prevents the full transformation of material into the desired output. Since we conceive of $y$ as a material output whose units are of constant weight, it follows immediately that

$$
\left.F_{m}\right|_{\left(\ell_{y}, m_{y}\right)}<1 \text { for } m_{y}=0 \text { and } \ell_{y} \geq 0 \text {. }
$$

When this property of $F$ is combined with the (conventional) assumption $F_{m m}<0$, we conclude that $F_{m}<1$ holds on the entire domain of the function $F{ }^{7}$

From the preceding discussion it is obvious that $y=F\left(\ell_{y}, m_{y}\right)$ is not a complete description of the production technology. After all, $F$ allows us to maintain some level (and weight) of output $y$ while varying the amount of material input. A minimum requirement for satisfying

\footnotetext{
${ }^{6}$ Note that the materials-balance principle also implies $\int_{0}^{\ell y} F_{\ell}\left(\lambda_{y}, m_{y}\right) d \lambda_{y} \leq m_{y}$ for all $m_{y} \geq 0$, since the output is made up of material $m_{y}$ only and the weight per unit of output $y$ is constant. This constraint is compatible with the assumptions $Y_{\ell}>0$ and $Y_{\ell \ell}<0$ if and only if $\lim _{\ell_{y} \rightarrow \infty} F_{\ell \ell}=0$.

${ }^{7}$ The upper bound $F_{m}<1$ is usually absent in textbook treatments of production functions, and it is violated, in particular, by the popular Cobb-Douglas function.
} 
the materials-balance principle is the existence of at least one more output, say a production residual $r_{y}$, to fill the materials-balance gap:

$$
\left.r_{y}+F\left(m_{y}, \ell_{y}\right)=m_{y} \quad \text { (and } r_{y} \geq 0\right) \text {. }
$$

In other words, bringing the conventional non-linear production function $y=F\left(\ell_{y}, m_{y}\right)$ in line with the materials-balance principle requires to look at production as transforming the material input, $m_{y}$, into two distinct outputs: the consumer good, $y$, whose generation is the purpose of the activity, and some production residuals, considered unwanted and environmentally harmful. From $r_{y}=m_{y}-F\left(m_{y}, \ell_{y}\right)$ follows $\left(d r_{y} / d m_{y}\right)=1-F_{m}$ which is positive due to the assumption $\left.F_{m} \in\right] 0,1[$.

At this point, production and abatement need to be linked up. Without any abatement, the total amount of production residuals generated, $r_{y}=m_{y}-F\left(m_{y}, \ell_{y}\right)$, would be discharged into the environment. But one can also hold back part of the production residuals from discharging, say the amount $a$, for abatement such that only the amount $e=r_{y}-a \geq 0$.

To sum up, the combined technologies of production and abatement are given by

$$
\begin{array}{lll}
y \leq F\left(\ell_{y}, m_{y}\right) & \text { (2a) } & \ell_{a}+\ell_{y}=\ell \\
r_{y}=m_{y}-y & \text { (2b) } & m_{a}+m_{y}=m \\
a \leq A\left(\ell_{a}, m_{a}\right) & \text { (2c) } & r_{a_{1}}=a \\
e=r_{y}-a & \text { (2d) } & r_{a_{2}}=m_{a}
\end{array}
$$

The properties $(A)$ and $(F)$ are satisfied

As discussed in the introduction, environmental economists have always been serious about joint production of wanted and unwanted outputs, about environmental damage caused by the emission of the latter and about residuals abatement to reduce emissions. But rather than focusing on comprehensive production-cum-abatement technologies such as (2), many of them used to employ production functions of type (1), i. e. the functional form $y=Y(e, \ell, m)$, where $y, e, \ell$ and $m$ are defined in the same way as in (2).

The comparison between (1) and (2) readily confirms that if the production function of type (1) is at all compatible with the production-cum-abatement technology (2) it is an incomplete 
description of that technology (2). The intriguing question is, therefore, what the precise relationship is between (1) and (2). ${ }^{8}$ Can a function of type (1) with property (Y) be shown to be implied by (2) or is (1) incompatible with (2)? In the latter case, one would need to discard the technology (1) since the materials-balance principle cannot be dispensed with.

To see what the link between (1) and (2) is like we now scrutinize the comprehensive technology (2) to elicit its major properties in several steps.

\section{Proposition 1:}

(i) Define $\Upsilon:=\left\{(y, e, \ell, m) \in \mathbb{R}^{4} \mid z=z(v)\right.$ for $z=y, e, \ell, m$ and $\left.v \in \Omega\right\}$, where $z(v)$ is the component $z$ of vector $v=\left(a, e, \ell, \ell_{a}, \ell_{y}, m, m_{a}, m_{y}, r_{a_{1}}, r_{a_{2}}, r_{y}, y\right) \in \mathbb{R}_{+}^{12}$ and where $\Omega:=\{v \mid v$ satisfies $(2 a)-(2 i)\}$. The set $\Upsilon$ is convex..$^{9}$

(ii) If (2a) and (2b) hold as equalities there is a mapping $G:(e, \ell, m) \rightarrow y$ implied by the production system (2) whose image is set valued.

To establish proposition 1(i), it suffices to prove convexity of the set $\Omega$, since if $\Omega$ is convex, its projection $\Upsilon$ into the subspace of all vectors $(y, e, \ell, m)$ is convex, too. Consider $\alpha \in[0,1], v^{\alpha}:=\alpha v^{1}+(1-\alpha) v^{2}$ and $v^{i}:=\left(a^{i}, e^{i}, \ell^{i}, \ell_{a}^{i}, \ell_{y}^{i}, m^{i}, m_{a}^{i}, m_{y}^{i}, r_{a_{i}}^{i}, r_{a_{2}}^{i}, r_{y}^{i}, y^{i}\right)$ for $i=$

1, 2. By definition, $\Omega$ is convex if for any pair $v^{1}, v^{2} \in \Omega$ it is true that $v^{\alpha} \in \Omega$ for all $\alpha \in[0,1]$. Hence we need to show that (2a) - (2i) is satisfied for $v^{\alpha}$. Consider $r_{y}^{\alpha}, m_{y}^{\alpha}, y^{\alpha}$ and confirm that these variables satisfy (2b) by calculating

$$
\begin{aligned}
m_{y}^{\alpha}-y^{\alpha} & =\alpha m_{y}^{1}+(1-\alpha) m_{y}^{2}-\alpha y^{1}-(1-\alpha) y^{2}=\alpha\left(m_{y}^{1}-y^{1}\right)+(1-\alpha)\left(m_{y}^{2}-y^{2}\right)= \\
& =\alpha r_{y}^{1}+(1-\alpha) r_{y}^{2}=r_{y}^{\alpha}
\end{aligned}
$$

Quite obviously, the same procedure can be applied to all linear equations in (2). It therefore only remains to show that (2a) and (2c) are satisfied for $v^{\alpha}$. This is easily established by observing that $y^{\alpha}=\alpha y^{1}+(1-\alpha) y^{2} \leq \alpha F\left(\ell_{y}^{1}, m_{y}^{1}\right)+(1-\alpha) F\left(\ell_{y}^{2}, m_{y}^{2}\right) \leq F\left(\ell_{y}^{\alpha}, m_{y}^{\alpha}\right)$, where the last inequality is due to the assumption that $F$ is a concave function. Since the function $A$ is also assumed concave, convexity of $\Omega$ is proved.

\footnotetext{
8 An early discussion on the relationship between technological concepts similar to (1) and (2) can be found in Siebert et al. (1980), where the technology of type (1) is referred to as net-emissions approach and that of type (2) as gross-emissions approach. Yet the technological concepts employed in Siebert et al. (1980) are not in line with the materials-balance principle. See also Pethig (2003).

${ }^{9}$ The sets $\Omega$ and $\Upsilon$ are also closed since (2) doesn't contain inequalities excluding the equality sign.
} 
We now turn to proving proposition 1(ii). Let (2a) and (2c) hold as equalities and consider (2a), (2c) and (2d) - (2f) to turn (2b) into $m_{y}=e+A\left(\ell-\ell_{y}, m-m_{y}\right)+F\left(\ell_{y}, m_{y}\right)$. Total differentiation of this equation yields, after some rearrangement of terms,

$$
d m_{y}=\frac{1}{1+A_{m}-F_{m}} d e+\frac{A_{\ell}}{1+A_{m}-F_{m}} d \ell-\frac{A_{\ell}-F_{\ell}}{1+A_{m}-F_{m}} d \ell_{y}+\frac{A_{m}}{1+A_{m}-F_{m}} d m .
$$

Next we set $d e=d \ell=d m=0$ in (4), totally differentiate (2a) and (2c) to obtain, under consideration of (2e) and (2f),

$$
\begin{aligned}
& d y=F_{\ell} d \ell_{y}+F_{m} d m_{y}=\frac{F_{\ell}\left(1+A_{m}\right)-F_{m} A_{\ell}}{1+A_{m}-F_{m}} d \ell_{y} \geq 0, \\
& d a=-A_{\ell} d \ell_{y}-A_{m} d m_{y}=-\frac{A_{m} F_{\ell}+A_{\ell}\left(1-F_{m}\right)}{1+A_{m}-F_{m}} d \ell_{y}<0 \text { and hence } \\
& \frac{d y}{d a}=\frac{F_{m} A_{\ell}-F_{\ell}\left(1+A_{m}\right)}{A_{m} F_{\ell}+A_{\ell}\left(1-F_{m}\right)} \geq 0 .
\end{aligned}
$$

Since $\left.F_{m} \in\right] 0,1\left[\right.$ and $A_{m}>0$ by assumption we have $1+A_{m}-F_{m}>0$. However, the numerator on the right side of (4a) may attain either sign so that $y$ may increase or decrease by shifting inputs from production, $\left(\ell_{y}, m_{y}\right)$, to abatement, $\left(\ell_{a}, m_{a}\right)$, while leaving net emissions $e$ unchanged. Equation (4c) demonstrates that for given $(e, \ell, m)$ shifting the inputs labor and material between production and abatement affects both the amount of production residuals abated and the amount of consumer goods produced. The sign of $d y / d a$ in (4c) is unclear.

The message of proposition 1(i) is that if there is a function of type (1) implied by (2) it will be concave. Unfortunately, proposition 1(ii) informs us that there is a correspondence rather than a function. Yet this lack of uniqueness can be overcome in a natural way since we are interested, of course, in the level of abatement which, for any given $(e, \ell, m)$, yields the maximum possible amount of the wanted output. That particular level of abatement will be called efficient. To characterize the efficient abatement we maximize with respect to $a, \ell_{a}$, $\ell_{y}, m_{a}, m_{y}$ and $r_{y}$ the wanted output $F\left(\ell_{y}, m_{y}\right)$ subject to (2b) - (2f). The associated Lagrangean reads

$$
\begin{aligned}
L= & y+\lambda_{y}\left[F\left(\ell_{y}, m_{y}\right)-y\right]+\lambda_{a}\left[A\left(\ell_{a}, m_{a}\right)-a\right]+\lambda_{e}\left[e+a+y-m_{y}\right] \\
& +\lambda_{\ell}\left[\ell-\ell_{a}-\ell_{y}\right]+\lambda_{m}\left[m-m_{a}-m_{y}\right]
\end{aligned}
$$


where $\lambda_{y}, \lambda_{a}, \lambda_{e}, \lambda_{\ell}$ and $\lambda_{m}$ are Lagrangean multipliers. Since the objective function is linear and all terms in the cornered brackets are concave functions the Kuhn-Tucker conditions are necessary and sufficient for a maximum. An interior solution implies

$$
\frac{F_{\ell}}{F_{m}} \cdot\left(1+A_{m}\right)=A_{\ell} \text { as well as } A_{\ell}>F_{\ell} \text {. }
$$

Note that (5) also follows from setting $d y / d a=0$ in (5c). $A_{\ell}>F_{\ell}$ follows from rearranging the equation in (5): $F_{\ell}\left(1+A_{m}-F_{m}\right)=F_{m}\left(A_{\ell}-F_{\ell}\right)>0$.

With the concept of efficient abatement we now continue our inquiry into the relationship between the production system (2) and the production function (1).

Proposition 2: If abatement is efficient, the production-cum-abatement technology (2) implies a production function $Y: D \rightarrow \mathbb{R}_{+}$that exhibits the properties $\left(Y^{*}\right)$ defined as

(a) Y exhibits the properties (Y),

(b) Y satisfies $\left.Y_{m} \in\right] 0, F_{m}\left[\right.$ [where $\left.F_{m} \in\right] 0,1[$ due to properties $(F)]$,

(c) The domain of $Y$ is $D:=\{(e, \ell, m) \mid e \leq m-F(\ell, m)\} \subset \mathbb{R}_{+}^{3}$;

Proposition 2 will now be proved in six steps.

Step 1: If abatement is efficient, the set of equations (2a) - (2f) implies a function $M:(e, \ell, m) \rightarrow m_{y}$ such that ${ }^{10}$

$$
m_{y}=M(e, \ell, m)
$$

Rewrite (5a) as $F_{\ell}\left(1+A_{m}\right)=A_{\ell} F_{m}$, totally differentiate this equation and combine the result with $d \ell=d \ell_{a}+d \ell_{y}$ and $d m=d m_{a}+d m_{y}$ from (2e) and (2f). After some rearrangements, these operations result in

$$
\begin{aligned}
& d \ell_{y}=\frac{\gamma_{m_{y}}}{\gamma_{\ell_{y}}} d m_{y}+\frac{\gamma_{\ell}}{\gamma_{\ell_{y}}} d \ell-\frac{\gamma_{m}}{\gamma_{\ell_{y}}} d m, \\
& \text { where } \quad \gamma_{\ell_{y}}:=A_{\ell} F_{m \ell}+F_{\ell} A_{m \ell}-\left(1+A_{m}\right) F_{\ell \ell}-F_{m} A_{\ell \ell}>0, \gamma_{\ell}:=F_{\ell} A_{m \ell}-F_{m} A_{\ell \ell}>0, \\
& \gamma_{m_{y}}:=F_{m} A_{\ell m}+\left(1+A_{m}\right) F_{\ell m}-F_{\ell} A_{m m}-A_{\ell} F_{m m}>0 \text { and } \gamma_{m}:=F_{m} A_{\ell m}-F_{\ell} A_{m m}>0 .
\end{aligned}
$$

\footnotetext{
${ }^{10}$ A plus or minus sign underneath an argument of a function indicates the (assumed) sign of the corresponding first partial derivative.
} 
Next we insert $d \ell_{y}$ from (7) into (3):

$d m_{y}\left(1+A_{m}-F_{m}\right)=d e+A_{\ell} d \ell+A_{m} d m-\frac{\left(A_{\ell}-F_{\ell}\right) \gamma_{m_{y}}}{\gamma_{\ell_{y}}} d m_{y}-\frac{\left(A_{\ell}-F_{\ell}\right) \gamma_{\ell}}{\gamma_{\ell_{y}}} d \ell+\frac{\left(A_{\ell}-F_{\ell}\right) \gamma_{m}}{\gamma_{\ell_{y}}} d m$.

Rearranging this equation yields

$d m_{y}=\frac{1}{\pi_{m 1}} d e+\frac{\pi_{m \ell}}{\pi_{m 1}} d \ell+\frac{\pi_{m m}}{\pi_{m 1}} d m$,

where $\pi_{m 1}=\left(1+A_{m}-F_{m}\right) \gamma_{\ell_{y}}+\left(A_{\ell}-F_{\ell}\right) \gamma_{m_{y}}>0, \pi_{m m}=A_{m} \gamma_{\ell_{y}}+\left(A_{\ell}-F_{\ell}\right) \gamma_{m}>0$

and $\quad \pi_{m \ell}=A_{\ell} \gamma_{\ell_{y}}-\left(A_{\ell}-F_{\ell}\right) \gamma_{\ell}=\left(A_{\ell}+F_{\ell}\right) \gamma_{\ell}+\left[A_{\ell} F_{m \ell}-\left(1+A_{m}\right) F_{\ell \ell}\right] A_{\ell}>0$.

Obviously, equation (8) determines the first derivatives of a function $M:(e, \ell, m) \rightarrow m_{y}$. Hence (6) is established.

Step 2: If abatement is efficient, the set of equations (2a) - (2f) implies a function $L:(e, \ell, m) \rightarrow \ell_{y}$ such that

$$
\begin{gathered}
\ell_{y}=L(e, \ell, m) . \\
++?
\end{gathered}
$$

$d m_{y}$ from (3) is now plugged into (7) yielding after some calculations

$$
d \ell_{y}=\frac{\gamma_{m_{y}}}{\pi_{m 1}} d e+\frac{\rho_{\ell}}{\pi_{m 1}} d \ell+\frac{\rho_{m}}{\pi_{m 1}} d m
$$

where $\rho_{\ell}:=\left(1+A_{m}-F_{m}\right) \gamma_{\ell}+A_{\ell} \gamma_{m_{y}}>0$ and $\rho_{m}:=\left(1-F_{m}\right) \gamma_{m}-\left[\left(1+A_{m}\right) F_{\ell m}-A_{\ell} F_{m m}\right] A_{m}$.

In view of (10) there is a function $L:(e, \ell, m) \rightarrow \begin{gathered}\ell_{y} \\ \text {, satisfying } \ell_{y}=L(e, \ell, m) . \\ ++?\end{gathered}$

Step 3: The preceding steps 1 and 2 imply a production function $Y:(e, \ell, m) \rightarrow y$ satisfying $Y_{e}>0, Y_{\ell}>0$ and $\left.Y_{m} \in\right] 0, F_{m}[$.

Invoking (6) and (9) the production function (2a) is turned into

$$
y=F\left[\begin{array}{c}
L(e, \ell, m), M(e, \ell, m)] \\
++?+Y(e, \ell, m)
\end{array}\right.
$$

The function $Y$ defined above obviously exhibits $Y_{e}>0$ and $Y_{\ell}>0$ but the sign of

$$
Y_{m}=F_{\ell} L_{m}+F_{m} M_{m}
$$


is unclear. To prove $\left.Y_{m} \in\right] 0, F_{m}[$, let (2a) and (2c) hold as equalities and combine the equations (2a) - (2f), (6) and (9) to rewrite (2b) as

$$
e+A[\ell-L(e, \ell, m), m-M(e, \ell, m)]+F[L(e, \ell, m), M(e, \ell, m)]=M(e, \ell, m) .
$$

We differentiate this equation with respect to $m$ (keeping $e$ and $\ell$ constant):

$$
-A_{\ell} L_{m}+A_{m}\left(1-M_{m}\right)+F_{\ell} L_{m}+F_{m} M_{m}=M_{m}
$$

which yields, after rearrangement of terms,

$$
L_{m}=\frac{A_{m}-\left(1+A_{m}-F_{m}\right) M_{m}}{A_{\ell}-F_{\ell}} .
$$

Next we substitute this term for $L_{m}$ in (11) and get

$$
Y_{m}=F_{\ell} L_{m}+F_{m} M_{m}=\frac{A_{m} F_{\ell}}{A_{\ell}-F_{\ell}}-\left[\frac{F_{\ell}\left(1+A_{m}-F_{m}\right)}{A_{\ell}-F_{\ell}}-F_{m}\right] M_{m}=\frac{A_{m} F_{\ell}}{A_{\ell}-F_{\ell}},
$$

since owing to (5) the term in the cornered brackets is zero. Moreover, $A_{\ell}, A_{m}$ and $F_{\ell}$ are positive due to the properties (A) and (F), and $A_{\ell}-F_{\ell}$ is positive due to (5). Therefore $Y_{m}=\frac{A_{m} F_{\ell}}{A_{\ell}-F_{\ell}}>0$. By invoking (5) again we find $Y_{m}=\frac{A_{m} F_{\ell}}{A_{\ell}-F_{\ell}}=F_{m}-\frac{F_{\ell}\left(1-F_{m}\right)}{A_{\ell}-F_{\ell}}<F_{m}$.

Step 4: The production function $Y$ from (11) is concave.

In view of the complex terms constituting its first derivatives there is no way to further specify its curvature by determining the sign of its second-order derivatives. Yet concavity of $Y$ is straightforward from the convexity of the set $\Upsilon$ that was established in proposition 1(i).

Step 5: The domain of the production function $Y$

We now determine the domain $D$ of function $Y$ by observing that (3) yields

$$
\frac{d e}{d m_{y}}=1+A_{m}-F_{m}>0 \text { and } \frac{d e}{d \ell_{y}}=A_{\ell}-F_{\ell}>0
$$

for given $\ell$ and $m$. That implies, in turn,

$$
\max _{\ell_{y}, m_{y}}\left[m_{y}-A\left(\ell-\ell_{y}, m-m_{y}\right)-F\left(\ell_{y}, m_{y}\right)\right]=m-A(0,0)-F(\ell, m)=m-F(\ell, m) .
$$

Consequently, for given inputs $\ell$ and $m$ the amount of production residuals emitted is largest when no abatement takes place at all. As an implication, the domain of $Y$ from (11) is $D$ as 
defined above. The upper bound which is placed on $e$ in $D$ is due to the fact that $e$ is an output and the law of mass conservation doesn't allow for an arbitrary expansion of a material output in a production process with a limited (finite) amount of material input. This completes the proof of proposition 2 .

Proposition 2 constitutes an important step toward reconciling the use of the production function (1) with material-balance requirements. It shows (i) that if efficient abatement is presupposed, the output of the consumer good is uniquely determined by $(e, \ell, m)$ and (ii) that the implied production function satisfies the properties $(\mathrm{Y})$ since it satisfies the properties $\left(\mathrm{Y}^{*}\right)$. Thus we confirmed that the properties $(\mathrm{Y})$ are necessary for any production function to be compatible with the technology (2). We also showed, however, that (2) imposes further constraints on the function $Y$ from (11) concerning its domain and the derivative $Y_{m}$. Both these additional restrictions can be easily violated if production functions $Y$ of type (1) are employed that exhibit the properties $(Y)$ only. In particular, $\left.Y_{m} \in\right] 0, F_{m}[$ implies that popular parametric production functions such as Cobb-Douglas functions don’t qualify for representing technologies of type (2).

In proposition 2 we didn't account for the residuals resulting from the abatement process. Now we make up for this omission in

\section{Proposition 3:}

(i) Provided that the abatement activity is always kept at an efficient level, the production-cum-abatement technology (2) is equivalent to three functions $Y, R^{1}$, and $R^{2}$ that map $(e, \ell, m) \in D$ into $Y(e, \ell, m), R^{1}(e, \ell, m)$ and $R^{2}(e, \ell, m)$, where $D$ and $Y$ are specified as in proposition 2, and where

$$
\begin{aligned}
& r_{a_{1}}=R^{1}(e, \ell, m):=A[\ell-L(e, \ell, m), m-M(e, \ell, m)] \geq 0, \\
& r_{a_{2}}=R^{2}(e, \ell, m):=T\left[R^{1}(e, \ell, m), \ell-L(e, \ell, m)\right] \geq 0, \text { and } \\
& T:\left(a, \ell_{a}\right) \rightarrow m_{a} \text { is a function such that } m_{a}=T\left(a, \ell_{a}\right) \text {, if and only if } a=A\left(\ell_{a}, m_{a}\right) .
\end{aligned}
$$

(ii) The functions $R^{1}$ and $R^{2}$ are concave. Its derivatives are indeterminate in sign except for $R_{e}^{1}<0$ and $R_{e}^{2}<0$. 
First we specify the functions $R^{1}$ and $R^{2}$ from (13) and (14), respectively. As for the abatement residuals of type 1 , we combine (2c), (2e) - (2g), (8) and (10) to obtain (13). Since $r_{a_{2}}=m_{a}$ due to (2h), we obtain $r_{a}=T\left(a, \ell_{a}\right)$ (with $T$ as defined in proposition 3(i)). When combined with (9), (13), (2e) and (2g), this equation yields (14). Concavity of the functions $R^{1}$ and $R^{2}$ follows immediately from the convexity of the set $\Omega$ established in the proof of proposition 1(i).

To see how the generation of abatement residuals reacts on the emission of production residuals we take the partial derivatives

$$
R_{e}^{1}=-\left(A_{\ell} L_{e}+A_{m} M_{e}\right) \quad \text { and } \quad R_{e}^{2}=-\left[T_{a} A_{m} M_{e}+\left(T_{a} A_{\ell}+T_{\ell}\right) L_{e}\right]=-M_{e} .
$$

While $R_{e}^{1}<0$ is obvious, $R_{e}^{2}=-M_{e}<0$ is explained by observing that total differentiation of $a=A\left(\ell_{a}, m_{a}\right)$ yields $d m_{y}=\frac{1}{A_{m}} d a-\frac{A_{\ell}}{A_{m}} d \ell_{a}$ and that in view of the definition of function $T$ above we clearly have $T_{a}=\left(1 / A_{m}\right)$ and $T_{\ell}=-\left(A_{\ell} / A_{m}\right)$. The signs of the partial derivatives $R_{e}^{1}$ and $R_{e}^{2}$ are as expected: If the scale of abatement is stepped up (such that the emissions $e$ are reduced) more abatement residuals are generated and vice versa. Checking the remaining partial derivatives of (13) and (14) reveals that their sign is ambiguous. This indeterminacy demonstrates that the impact of $\ell$ and $m$ on $r_{a_{1}}$ and $r_{a_{2}}$ is quite complex in spite of the simple hypotheses (2g) and (2h).

In view of the propositions 2 and 3 the technology (2) is completely and compactly described by

$$
y=Y(e, \ell, m), r_{a_{1}}=R^{1}(e, \ell, m) \text { and } r_{a_{2}}=R^{2}(e, \ell, m) .
$$

where $Y, R^{1}$ and $R^{2}$ are as defined in (11), (13) and (14), respectively. In proposition 2 the function $Y$ from (11) has been shown to satisfy properties $\left(\mathrm{Y}^{*}\right)$ while the curvature of $R^{1}$ and $R^{2}$ is less well known (proposition 3(ii)). With proposition (2) our main goal is clearly achieved, namely to show that the properties (Y) are necessary conditions for functions (1) to represent a subsystem of the comprehensive technology (2). In proposition (2) we even proved that the properties $(\mathrm{Y})$ are not sufficient since the properties $\left(\mathrm{Y}^{*}\right)$ are more restrictive than the properties (Y). 
Yet the characterization of the relationship between (1) and (2) is still incomplete from a theoretical point of view. One may want to know which the comprehensive set of conditions is that must be satisfied by the functions $Y, R^{1}$ and $R^{2}$ from (15) to secure that these functions are equivalent to some technology (2). Unfortunately there is little hope to make progress toward this end because the properties of $Y, R^{1}$ and $R^{2}$ from (15) are made up of a complex mix of first and higher-order derivatives of the functions $A$ and $F$ from (2). The basic production functions $A$ and $F$ determine the curvature of $Y, R^{1}$ and $R^{2}$ in a very complex and interdependent way involving a constrained maximization procedure (efficient abatement).

It is true that without a complete set of conditions we cannot decide whether a given conventional production function (1) is compatible with the comprehensive technology (2) or not. Yet the value of such a result is not so clear. The information provided in propositions 2 and 3 appears to be sufficient for most theoretical modeling exercises, since in those studies first and higher-order derivatives are usually not quantitatively (let alone numerically) specified. For applied research, it is hardly appropriate to start out with some 'arbitrary' function $Y$ satisfying the properties $\left(\mathrm{Y}^{*}\right)$. One would rather have to start with the empirically valid specification of the true technology (2) anyway, since (11), (13) and (14) are and always will be derived from the true empirically observable technology (2).

\section{Allocative efficiency and materials balance in an economy with production, abate- ment and pollution}

We now envisage a simple economy where an (aggregate) firm applies technology (2). The consumption of the only wanted output $y$ is modeled as a process of material transformation like production and abatement: it consists in turning the amount $y$ of the consumer good into the amount $r_{c}$ of a post-consumption residual. Both are equal in weight,

$$
y=r_{c} .
$$

Thus we now deal with four different types of residuals: $e, r_{a 1}, r_{a 2}$ and $r_{c}$, each of which has the potential to degrade the environment after having been discharged. Denote by $x$ an index of the ambient concentration of pollutants, called pollution for short. We define

$$
x=X\left(e, r_{a_{1}}, r_{a_{2}}, r_{c}\right) \quad \text { with } X(0,0,0,0)=0
$$


as the pollution that results from releasing the residuals $e, r_{a 1}, r_{a 2}$ and $r_{c}$ into the environment. $X$ is assumed to be non-decreasing and convex. If $X_{v}=0$ for $v=r_{a 1}, r_{a 2}$, or $r_{c}$ the level of the residual under consideration is small enough to be fully neutralized by nature's assimilative capacity. Environmental degradation as described by $x$ negatively affects the consumers' utility

$$
u_{i}=U^{i}\left(x, y_{i}\right) \quad i=1, \ldots, n
$$

The general equilibrium model is completed by introducing the standard resource constraints

$$
y \geq \sum_{i} y_{i}, \quad \bar{\ell} \geq \ell \quad \text { and } \quad \bar{m} \geq m,
$$

where $\bar{\ell}$ and $\bar{m}$ are the economy's fixed factor endowments. To characterize a Pareto efficient allocation of the economy (15) - (19) we solve the Lagrangean

$$
\begin{aligned}
& \sum_{i} \alpha_{i} U^{i}\left(x, y_{i}\right)+\lambda_{f}[Y(e, \ell, m)-y]+\lambda_{x}\left[x-X\left(e, r_{a 1}, r_{a 2}, r_{c}\right)\right]+\lambda_{1}\left[r_{a 1}-R^{1}(e, \ell, m)\right]+ \\
& +\lambda_{2}\left[r_{a 2}-R^{2}(e, \ell, m)\right]+\lambda_{y}\left(y-\sum_{i} y_{i}\right)+\lambda_{c}\left(r_{c}-y\right)+\lambda_{\ell}(\bar{\ell}-\ell)+\lambda_{m}(\bar{m}-m),
\end{aligned}
$$

where $\alpha_{i}$ are arbitrary positive numbers for $i=1, \ldots, n$. Under the assumption that an interior $^{11}$ solution exists the first-order conditions are

$$
\begin{aligned}
& \lambda_{y}=\alpha_{i} U_{y}^{i} \quad i=1, \ldots, n \\
& \lambda_{x}=-\sum_{i} \alpha_{i} U_{x}^{i} \\
& \lambda_{f} Y_{e}=\lambda_{x} X_{e}+\sum_{j} \lambda_{j} R_{e}^{j} \\
& \lambda_{f} Y_{\ell}=\lambda_{\ell}+\sum_{j} \lambda_{j} R_{\ell}^{j} \\
& \lambda_{f} Y_{m}=\lambda_{m}+\sum_{j} \lambda_{j} R_{m}^{j}
\end{aligned}
$$

Proposition 4: The efficient allocation of the economy (15) - (19) is characterized by

$$
M D_{x}=\frac{Y_{e}}{X_{e}} \cdot Q \text { with } M D_{x}:=-\sum_{i} \frac{U_{x}^{i}}{U_{y}^{i}} \text { and } Q:=\frac{\left(1+X_{r_{c}} \sum_{i} \frac{U_{x}^{i}}{U_{y}^{i}}\right) X_{e}}{X_{e}+\sum_{j} X_{r_{a j}} R_{e}^{j}} .
$$

As a consequence,

\footnotetext{
11 In the present context, an interior solution implies that $(e, \ell, m)$ is in the interior of the domain $D$ of function $Y$. This doesn't only require all variables to be bounded away from zero but also that the inequality $e \leq m-F(\ell, m)$ is not binding. For the sake of completeness one would have had to consider this inequality as an additional constraint in (20). But with the strict inequality sign in the solution the associated Lagrange multiplier would be zero and therefore need not be introduced in the first place.
} 


$$
M D_{x} \cdot X_{e}\{\gtreqless\} Y_{e} \text { if }\left\{\begin{array}{l}
X_{r_{a 1}}>0 \text { and } / \text { or } X_{r_{a 2}}>0 \text { and } X_{r_{c}}=0 \\
X_{r_{a 1}}=X_{r_{a 2}}=X_{r_{c}}=0, \\
X_{r_{a 1}}=X_{r_{a 2}}=0 \text { and } X_{r_{c}}>0 .
\end{array}\right.
$$

To derive (22) from (21), we first consider (21f) and (21g) to obtain $\frac{\lambda_{y}}{\lambda_{f}}=\frac{\lambda_{f}+\lambda_{c}}{\lambda_{f}}=$ $=1+\frac{\lambda_{x}}{\lambda_{f}} X_{r_{c}}$. Next we combine this equation with (21a) to turn (21b) into

$$
\frac{\lambda_{x}}{\lambda_{f}}=-\frac{\lambda_{y}}{\lambda_{f}} \sum_{i} \frac{U_{x}^{i}}{U_{y}^{i}}=-\left(1+\frac{\lambda_{x}}{\lambda_{f}} X_{r_{c}}\right) \sum_{i} \frac{U_{x}^{i}}{U_{y}^{i}}=-\frac{\sum_{i} \frac{U_{x}^{i}}{U_{y}^{i}}}{1+X_{r_{c}} \sum_{i} \frac{U_{x}^{i}}{U_{y}^{i}}}
$$

In (24), $\left.\left(1+X_{r_{c}} \sum_{i} \frac{U_{x}^{i}}{U_{y}^{i}}\right) \in\right] 0,1\left[\right.$, since $\lambda_{x} / \lambda_{f}>0$ in an interior solution. We proceed by inferring $\frac{\lambda_{x}}{\lambda_{f}}=\frac{Y_{e}}{X_{e}+\sum_{j} X_{r_{a j}} R_{e}^{j}}$ from (21c), (21h) and (21k). (22) is then obtained by inserting this equation into (24).

Clearly, (22) characterizes the efficient level of pollution generated jointly via the emission of all residuals. $M D_{x}$ is the damage from a marginal increase in pollution evaluated by the consumers' aggregate marginal willingness-to-pay in terms of the consumer good for avoiding that increase. The right side of (22) represents the marginal benefit of pollution that comes in form of an increase in the consumer good made possible by a small increase in pollution through stepping up emissions. Equivalently, one can interpret $M D_{x}$ as the marginal benefit and $Y_{e} Q / X_{e}$ as the marginal cost of reducing pollution. To further interpret the term $Y_{e} Q / X_{e}$ observe that $Y_{e}$ is the amount of consumer goods that cannot be produced anymore when the emission of production residuals is reduced by a small amount. It is known as marginal abatement costs of production residuals (in terms of the consumer good) in models where no residuals other than production residuals are considered. For convenience, we will refer to $Y_{e}$ as conventional marginal abatement costs of production residuals.

If we multiply both sides of (22) by $X_{e}$ we obtain $M D_{x} \cdot X_{e}=Y_{e} \cdot Q$. The left side of this equation represents the marginal benefit of reducing the emission of production residuals and 
the right side consists of the marginal abatement costs of production residuals. Those costs differ from $Y_{e}$ unless $Q=1$. In fact, inspection of the definition of $Q$ in (22) reveals that given $X_{e}>0$ (which we presuppose throughout) $Q$ may be greater or smaller than unity depending on which other residuals contribute to pollution. More specifically, the optimality condition $M D_{x} \cdot X_{e}=Y_{e} \cdot Q$ and the definition of $Q$ from (22) imply (23).

The striking result is that $Y_{e}$, the conventional marginal abatement cost of production residuals, is an incorrect measure of the social marginal abatement costs of production residuals, in general, when in addition to production residuals other residuals contribute to pollution, too. ${ }^{12}$ As shown in (23), if abatement residuals are pollutants in addition to production residuals but post-consumption residuals are not, $Y_{e}$ underestimates the true marginal abatement costs of production residuals. This is so because the abatement of production residuals generates a negative pollution externality through the increase of abatement residuals which it unavoidably brings about but which is not accounted for in $Y_{e}$. Conversely, if post-consumption residuals pollute in addition to production residuals but abatement residuals don't, we infer from (23) that $Y_{e}$ overestimates the (true) marginal abatement costs of production residuals since this time abatement induces a positive externality in form of a small reduction in pollution caused by post-consumption residuals which is not captured by $Y_{e}$.

\section{Corrective taxes in the competitive market economy}

Consider now the economy of Section 3 with perfectly competitive markets for labor, material and the consumer good and denote the associated prices by $p_{\ell}, p_{m}$ and $p_{y}$, respectively. Quite obviously, such a market economy fails to be efficient in the absence of environmental policy. We are therefore interested in exploring what kind of tax schemes is capable to restore efficiency. As is well-known, if we would replace in our model (15) by (1) and replace (17) by $x=X(e)$ with $X_{e}>0$, the pollution externality would be internalized by the timehonored Pigouvian tax rule according to which the tax rate $t_{e}$ on emissions $e$ is to be set equal to the conventional marginal abatement cost, $Y_{e}$. But in our model with the comprehensive production-cum-abatement technology (2) it is also interesting to investigate the deviations

\footnotetext{
12 The added term 'in general' weakens that statement slightly. Yet it is necessary since marginal pollution effects of abatement residuals and post-consumption residuals are opposite in sign. It cannot be excluded, therefore, that both effects compensate each other incidently.
} 
from that conventional Pigouvian tax rule required in a model containing (15) and (17) and when $X_{r a 1}, X_{r a 2}$ and/or $X_{r c}$ are positive.

To address this issue we need to look at the agents' optimization problems as implicitly described by the Lagrangeans ${ }^{13}$

$$
\begin{aligned}
& U^{i}\left(x, y_{i}\right)+\mu_{i}\left[p_{\ell} \bar{\ell}_{i}+p_{m} \bar{m}_{i}+\theta_{i}(g+b)-p_{y} y\right] \text { and } \\
& \left\{p_{y} y-\left(p_{\ell}+t_{\ell}\right) \ell-\left(p_{m}+t_{m}\right) m-t_{a 1} r_{a 1}-t_{a 2} r_{a 2}-t_{c} r_{c}-t_{e} e\right\}+\mu_{c}\left(r_{c}-y\right)+ \\
& +\mu_{f}[Y(e, \ell, m)-y]+\mu_{1}\left[r_{a 1}-R^{1}(e, \ell, m)\right]+\mu_{2}\left[r_{a 2}-R^{2}(e, \ell, m)\right] .
\end{aligned}
$$

With regard to post-consumption residuals, implicit in (25) and (26) is the so-called take-back rule as modeled, e. g., in Eichner and Pethig (2000). Each consumer $i$ purchases the amount $y_{i}$ of consumer goods, and after consumption she returns the post-consumption residuals to the producers. By institutional design, producers are responsible for the (orderly) deposition of these residuals, and they may be therefore charged an emission fee, $t_{c}$, if the postconsumption residuals cause pollution after having been emitted. This institutional arrangement explains why post-consumption residuals don't enter the consumers' optimization calculus (25), ${ }^{14}$ but rather are part of the (aggregate) firm's profit maximization calculus.

The (aggregate) firm employs the technology (15) and is charged input taxes, $t_{\ell}$ and $t_{m}$, as well as emission taxes, $t_{e}, t_{a 1}, t_{a 2}$ and $t_{c}$. Some of these tax rates may be zero, of course. The first-order conditions associated to an (interior) solution to (25) and (26) are conveniently summarized in

$$
\begin{array}{lll}
\mu_{i} p_{y}=U_{y}^{i}, & \text { (27a) } & \left(p_{y}-t_{c}\right) Y_{\ell}=p_{\ell}+t_{\ell}+\sum_{j} t_{a j} R_{\ell}^{j}, \\
\left(p_{y}-t_{c}\right) Y_{e}=t_{e}+\sum_{j} t_{a j} R_{e}^{j}, & \text { (27b) } & \left(p_{y}-t_{c}\right) Y_{m}=p_{m}+t_{m}+\sum_{j} t_{a j} R_{m}^{j} .
\end{array}
$$

\section{Proposition 4:}

\footnotetext{
13 For $v=\ell, m$, consumer i's factor endowment is $\bar{v}_{i}$ with $\sum_{i} \bar{v}_{i}=\bar{v} \cdot \theta_{i}$ is her share in the firm's profit, $g$, and the government's budget surplus, $b$. The consumers' profit and surplus shares satisfy $\sum_{j} \theta_{j}=1$.

14 One can easily account for that alternative by modifying (25) and (26) in the following way: In (25), add the term " $-t_{c} r_{C_{i}}$ " to the second expression in brackets and add the Lagrange constraint " $\lambda_{c_{i}} r_{C_{i}}\left(r_{c_{i}}-y_{i}\right)$ " at the end. In (26), delete the term " $-t_{c} r_{c}$ " and the Lagrange constraint " $\mu_{c}\left(r_{c_{i}}-y\right)$ ". In the absence of institution-specific costs the conditions for allocative efficiency are the same in both regimes (Eichner and Pethig 2000).
} 
(i) Suppose, that $\lambda_{1}, \lambda_{2}, \lambda_{c}, \lambda_{f}, \lambda_{\ell}, \lambda_{m}, \lambda_{x}$ and $\lambda_{y}$ are the values attained by the Lagrange multipliers in the solution to (20) and that the partial derivatives $X_{e}$ and $R_{h}^{j}$ for $j=1,2$ and $h=\ell, m$ are also evaluated at that solution. Set prices $p_{\ell}=\lambda_{\ell}, p_{m}=\lambda_{m}, p_{y}=\lambda_{y}$ and consider the alternative tax schemes $A$ and $B$ :

- A is defined by: $t_{a 1}^{A}=\lambda_{1}, t_{a 2}^{A}=\lambda_{2}, t_{c}^{A}=\lambda_{c}, t_{e}^{A}=\lambda_{x} X_{e}$ and $t_{\ell}^{A}=t_{m}^{A}=0$;

- $B$ is defined by: $\quad t_{a 1}^{B}=t_{a 2}^{B}=0, t_{c}^{B}=\lambda_{c}, t_{e}^{B}=\lambda_{x} X_{e}+\sum_{j} \lambda_{j} R_{e}^{j}, t_{\ell}^{B}=\sum_{j} \lambda_{j} R_{e}^{j}$ and $t_{m}^{B}=\sum_{j} \lambda_{j} R_{m}^{j}$.

With these prices and either tax scheme A or tax scheme B all markets clear and the equilibrium allocation is efficient.

(ii) When the tax scheme $A$ is implemented, the efficient tax rates satisfy

$$
t_{e}^{A}\{\gtreqless\} Y_{e} \text { if }\left\{\begin{array}{l}
X_{r_{a 1}}>0 \text { and } / \text { or } X_{r_{a 2}}>0 \text { and } X_{r_{c}}=0, \\
X_{r_{a 1}}=X_{r_{a 2}}=X_{r_{c}}=0, \\
X_{r_{a 1}}=X_{r_{a 2}}=0 \text { and } X_{r_{c}}>0 .
\end{array}\right.
$$

To prove Proposition 4i insert the prices and tax rates as assigned in Proposition 4 into (27) and verify that this substitution makes (27) coincide with (21). The tax scheme $A$ in Proposition $4 \mathrm{i}$ is a pure emissions tax scheme in the sense that a tax is levied on the emission of each polluting residual, while non-emission items like the inputs labor and material are not taxed. We infer from (21) that (in equilibrium) with the tax scheme $A$ all tax rates are set equal to the marginal environmental damage of the respective residuals ${ }^{15}$ :

$$
t_{v}^{A}=M D_{x} X_{v}:=-\sum_{i} \frac{U_{x}^{i} X_{v}}{U_{y}^{i}} \text { for } v=e, r_{a_{1}}, r_{a_{2}}, c
$$

We also know that if abatement takes place, optimal emissions tax rates need to equal marginal abatement costs. In our model production residuals are the only residuals subject to abatement. Therefore we will now focus on those residuals. Invoking (27b) we obtain, after some rearrangement of terms,

$$
t_{e}^{A}=Y_{e}-\sum_{j} t_{a j}^{A} R_{e}^{j}-t_{c}^{A} Y_{e}
$$

\footnotetext{
15 To simplify the comparison of marginal conditions characterizing either efficient allocations or market allocations we measure all prices and tax rates in terms of the consumer good by setting $p_{y} \equiv 1$.
} 
Maintaining the assumption $X_{e}>0$ (which implies $t_{e}^{A}>0$ owing to (29)) we now wish to juxtapose alternative scenarios where one, two or all of the (efficient!) tax rates $t_{a 1}^{A}, t_{a 2}^{A}$ and $t_{c}^{A}$ may be zero. In view of $Y_{e}>0, R_{e}^{1}<0$ and $R_{e}^{2}<0$, it is straightforward that (29) and (30) imply (28). (28) complements (24), in fact, but (28) was derived via (30) rather than via (23) and thus offers insights in the determinants of the efficient emissions tax rate $t_{e}$.

Suppose first, pollution is caused by the emission of production residuals only $\left(X_{r a 1}=X_{r a 2}=X_{r c}=0\right)$. Then $t_{e}=Y_{e}$ is optimal due to (28), i. e. the tax rate $t_{e}$ is required to be set equal to the conventional marginal costs of abating production residuals. When combined with (29) $t_{e}=Y_{e}$ yields

$$
-\sum_{i} \frac{U_{x}^{i} X_{e}}{U_{y}^{i}}=t_{e}=Y_{e}
$$

which constitutes the conventional Pigouvian tax rule. Yet in view of possible deviations from that rule, as described in (29) and (31), (31) is not a trivial result because we specified conditions under which the conventional Pigouvian tax rule is valid.

Suppose next that $X_{r_{a 1}}>0$ and/or $X_{r_{a 2}}>0$ but $X_{r_{c}}=0$. In this case the social marginal abatement costs of production residuals are $Y_{e}-\sum_{j} t_{a j}^{A} R_{e}^{j}>Y_{e}$. The extra marginal costs, $\sum_{j} t_{a j} R_{e}^{j}$, accrue because an increase in the abatement of production residuals inadvertently raises the generation of environmentally harmful abatement residuals. The result $t_{e}^{A}>Y_{e}$ is worth noting: It is now optimal to abate less production residuals than under the conventional Pigouvian tax rule. Conversely, if abatement residuals don't degrade the environment but post-consumption residuals do $\left(X_{r_{a 1}}=X_{r_{a 2}}=0\right.$ and $\left.X_{r_{c}}>0\right)$, then the social marginal abatement costs of production residuals are $Y_{e}-t_{c}^{A} Y_{e}<Y_{e}$. The extra marginal cost savings, $t_{c}^{A} Y_{e}$, occur because an increase in the abatement of production residuals inadvertently reduces the amount of post-consumption residuals and consumer goods. Since post-consumption residuals are (now) pollutants by assumption, curbing $r_{c}$ reduces that pollution which is, in turn, a beneficial external effect of abating production residuals. With $t_{e}^{A}<Y_{e}$ it is now optimal to abate more production residuals than under the conventional Pigouvian tax rule. 
Despite our main focus on the discussion of (28) one needs to keep in mind that according to (29) the efficient tax scheme A requires to levy a tax on all polluting residuals. It doesn't follow from setting $t_{e}^{A} \neq Y_{e}$ as prescribed by (28) that no emissions tax other than $t_{e}$ is needed to achieve efficiency in case that the production residuals are not the only pollutants. For that scenario our analysis provides an important lesson: If all polluting residuals are taxed in an effort to correct for the allocative distortion, it is not efficient, in general, to set the tax rate of the production residuals equal to their marginal abatement cost, conventionally defined.

Turning to the interpretation of tax scheme $B$ we observe that $B$ is also capable of restoring efficiency in the market economy and does so without any tax on abatement residuals. This scheme is a particularly interesting option for efficient pollution control if abatement residuals are difficult and costly to monitor and therefore cannot readily be used as a tax base. However, avoiding taxes on abatement residuals comes at the price of taxing labor and material (in addition to post-consumption and production residuals). ${ }^{16}$ Since the signs of the derivatives $R_{\ell}^{j}$ and $R_{m}^{j}$ for $j=1,2$ are ambiguous, it is not clear, whether $t_{m}$ and $t_{\ell}$ are subsidies or taxes (proper). At any rate, securing efficiency by means of tax scheme $B$ requires to drive tax wedges between demand prices and supply prices on all three markets. Taking a closer look at $t_{e}$ under tax scheme $B$ shows that

$$
-\sum_{i} \frac{U_{x}^{i}}{U_{y}^{i}}\left(X_{e}+\sum_{j} X_{r_{a j}} R_{e}^{j}\right)=t_{e}^{B}=Y_{e}-t_{c}^{B} Y_{e} .
$$

To the left of the first equality sign in (32) we have the sum of the direct (positive) and indirect (negative) marginal benefits of a small reduction in the emission of production residuals. The indirect benefit is, in fact, the marginal environmental damage from the emission of abatement residuals caused by stepping up abatement. Correspondingly, the far right side of (32) represents the social marginal abatement cost which is the same as in tax scheme $A$ for the case that $X_{r a 1}=X_{r a 2}=0$. Since $t_{e}^{B}<Y_{e}$, it is optimal to abate more production residuals than under the conventional Pigouvian tax rule.

\section{Concluding remarks}

\footnotetext{
16 One may wonder why there isn't a third efficiency restoring tax scheme, that taxes also labor and material (like $B$ ) but in which $t_{e}$ captures the impact of all kinds of residuals. After all, due to $r_{c}=y$, post-consumption residuals are generated uno actu with all other outputs. However, when we set $t_{a 1}=t_{a 2}=t_{c}=0$ in (26) there is no way to find values for $t_{\ell}, t_{m}$ and $t_{e}$ such that market equilibria turn out to be efficient.
} 
It is a standard procedure in environmental economics to model abatement as a non-linear technological process to reduce the discharge into the environment of a residual generated as a by-product of a wanted good. It is also standard in formal analyses of production-cumabatement to map incompletely the materials flow that is inevitably involved in that process. The present paper shows how the standard way of modeling production-cum-abatement can be brought into line with physical constraints securing materials balance. It demonstrates, in particular, that the production function (1) can be reconstructed from a comprehensive production-cum-abatement technology. Although (1) maps the materials flow incompletely, a production function of type (1) has been shown to be implied by each technology (2) that fully accounts for all materials flows involved. We have also been proved that the implied function $Y$ satisfies the properties $\left(\mathrm{Y}^{*}\right)$ that are sufficient but not necessary for the properties (Y). It remains an open question, therefore, whether for each function $Y$ satisfying the aforementioned properties $\left(\mathrm{Y}^{*}\right)$ there exists a technology (2) such that $Y$ is implied by that technology. Therefore we also don't know the comprehensive set of conditions that makes the functions $Y, R^{1}$ and $R^{2}$ from (15) fully compatible with the technology (2).

Moreover, in a simple general equilibrium model with full regard of the materials flow the paper explores the consequences for allocative efficiency and efficiency-restoring taxation of multiple interdependent pollutants that are inevitably linked to the transformation processes of production, abatement and consumption. Finally, the paper demonstrates that efficiency may require setting the emissions tax rate above or below conventionally defined marginal abatement cost if the residual subject to abatement is not the only residual causing environmental degradation.

Krysiak and Krysiak (2003) address the issue of modeling all processes of materials transformation consistently in an analytical framework that aims at maximum generality. We proceeded, instead, by trading generality for more specific and more informative results about the emergence and consequences of multiple and interdependent residuals and pollutants involved in production-cum-abatement when the materials-balance principle is explicitly and fully regarded.

As for the consequences of multiple residuals, we find, not surprisingly, that if the production residual, $r_{y}$, is the only pollutant it suffices to employ the 'truncated' production-cumabatement technology (1). However, as soon as the emission of at least one additional residual in the pertaining materials flow also contributes to the degradation of the environment, the need for an integrated multi-pollutant control arises (Guruswanny 1991). There is a growing 
awareness in both academia and the political arena (U.S. General Accounting Office 1996) that the most efficient control strategy is to consider multiple pollutants simultaneously rather than continue with the prevailing single-pollutant regulations. However, the demand for multi-pollutant control approaches is quite often rationalized by growing concerns about the potential risks to human health and/or to the environment from the interaction of multiple pollutants after their emission. These are certainly serious concerns in their own right. Yet the emphasis of the present paper is on allocative problems caused by multiple pollutants whose generation is interdependent. In that case, a first-best tax strategy also needs to account for the technical interdependencies among the residuals since such linkages impact on the efficient levels of all tax rates. As a consequence, the time-honored rule of equating the emissions tax rate and marginal abatement cost, defined in the conventional way, is shown to be no longer efficient, in general.

\section{References}

Ayres, Robert U., and Kneese, Allen V. (1969), "Production, consumption and externalities", American Economic Review 59, 282-97

Baumol, William J., and Oates, Wallace E. (1975), The Theory of Environmental Policy, Englewoods Cliffs, N. J.

Cropper, Maureen L., and Oates, Wallace E. (1992), "Environmental economics: A survey", Journal of Economic Literature 30, 675-740

Eichner, Thomas, and Pethig, Rüdiger (2000) "Recycling, producer responsibility and centralized waste management", Finanzarchiv 57, 333-360

Forster, B.A. (1972), "A note on economic growth and environmental quality", Swedish Journal of Economics 74, 281-285

Guruswanny, Lakshman (1991), "The case for integrated pollution control", Law and Contemporary Problems 54, 41-56

Klevorick, A.K., und Kramer, G.H. (1973), "Social choice on pollution management: The Genossenschaften", Journal of Public Economics 2, 101-146

Krysiak, Frank C., and Krysiak, Daniela (2003), "Production, consumption, and general equilibrium with physical constraints", Journal of Environmental Economics and Management, 46, 513-538

Mäler, Karl-Göran (1974), Environmental Economics: A Theoretical Inquiry, Baltimore

Pethig, Rüdiger (2003), "The 'materials-balance approach' to pollution: its origin, implications and acceptance", University of Siegen, Economics Discussion Paper No. 105-03

Pethig, Rüdiger (1975), "Umweltverschmutzung, Wohlfahrt und Umweltpolitik in einem Zwei-Sektoren-Gleichgewichtsmodell", Zeitschrift für Nationalökonomie 35, 99-124

Pezzey, John C. V., and Toman, Michael A. (2002), "Progress and problems in the economics of sustainability", in: T. Tietenberg and H. Folmer (eds.), The International Yearbook 
of Environmental and Resource Economics 2002/2003, Edward Elgar, Cheltenham, Northampton, 165-232

Siebert, Horst, Eichberger, Jürgen, Gronych Ralf, and Pethig, Rüdiger (1980), Trade and Environment: A Theoretical Inquiry, Elsevier, Amsterdam et al.

U.S. General Accounting Office (GAO) (1996), Environmental Management: An Integrated Approach Could Reduce Pollution and Increase Regulatory Efficiency, GAO/RCED96-41, U.S. GAO, Washington, D.C. 


\section{CESifo Working Paper Series}

(for full list see www.cesifo-group.de)

1485 Carsten Hefeker, Uncertainty, Wage Setting and Decision Making in a Monetary Union, June 2005

1486 Ondřej Schneider and Jan Zápal, Fiscal Policy in New EU Member States - Go East, Prudent Man!, June 2005

1487 Christian Schultz, Virtual Capacity and Competition, June 2005

1488 Yvan Lengwiler and Elmar Wolfstetter, Bid Rigging - An Analysis of Corruption in Auctions, June 2005

1489 Johannes Becker and Clemens Fuest, Does Germany Collect Revenue from Taxing Capital Income?, June 2005

1490 Axel Dreher and Panu Poutvaara, Student Flows and Migration: An Empirical Analysis, June 2005

1491 Bernd Huber and Marco Runkel, Interregional Redistribution and Budget Institutions under Asymmetric Information, June 2005

1492 Guido Tabellini, Culture and Institutions: Economic Development in the Regions of Europe, July 2005

1493 Kurt R. Brekke and Michael Kuhn, Direct to Consumer Advertising in Pharmaceutical Markets, July 2005

1494 Martín Gonzalez-Eiras and Dirk Niepelt, Sustaining Social Security, July 2005

1495 Alfons J. Weichenrieder, (Why) Do we need Corporate Taxation?, July 2005

1496 Paolo M. Panteghini, S-Based Taxation under Default Risk, July 2005

1497 Panos Hatzipanayotou and Michael S. Michael, Migration, Tied Foreign Aid and the Welfare State, July 2005

1498 Agata Antkiewicz and John Whalley, BRICSAM and the Non-WTO, July 2005

1499 Petr Hedbávný, Ondřej Schneider and Jan Zápal, A Fiscal Rule that has Teeth: A Suggestion for a 'Fiscal Sustainability Council' underpinned by the Financial Markets, July 2005

1500 J. Atsu Amegashie and Marco Runkel, Sabotaging Potential Rivals, July 2005 
1501 Heikki Oksanen, Actuarial Neutrality across Generations Applied to Public Pensions under Population Ageing: Effects on Government Finances and National Saving, July 2005

1502 Xenia Matschke, Costly Revenue-Raising and the Case for Favoring Import-Competing Industries, July 2005

1503 Horst Raff and Nicolas Schmitt, Why Parallel Trade may Raise Producers Profits, July 2005

1504 Alberto Bisin and Piero Gottardi, Efficient Competitive Equilibria with Adverse Selection, July 2005

1505 Peter A. Zadrozny, Necessary and Sufficient Restrictions for Existence of a Unique Fourth Moment of a Univariate GARCH(p,q) Process, July 2005

1506 Rainer Niemann and Corinna Treisch, Group Taxation, Asymmetric Taxation and Cross-Border Investment Incentives in Austria, July 2005

1507 Thomas Christiaans, Thomas Eichner and Ruediger Pethig, Optimal Pest Control in Agriculture, July 2005

1508 Biswa N. Bhattacharyay and Prabir De, Promotion of Trade and Investments between China and India: The Case of Southwest China and East and Northeast India, July 2005

1509 Jean Hindriks and Ben Lockwood, Decentralization and Electoral Accountability: Incentives, Separation, and Voter Welfare, July 2005

1510 Michelle R. Garfinkel, Stergios Skaperdas and Constantinos Syropoulos, Globalization and Domestic Conflict, July 2005

1511 Jesús Crespo-Cuaresma, Balázs Égert and Ronald MacDonald, Non-Linear Exchange Rate Dynamics in Target Zones: A Bumpy Road towards a Honeymoon - Some Evidence from the ERM, ERM2 and Selected New EU Member States, July 2005

1512 David S. Evans and Michael Salinger, Curing Sinus Headaches and Tying Law: An Empirical Analysis of Bundling Decongestants and Pain Relievers, August 2005

1513 Christian Keuschnigg and Martin D. Dietz, A Growth Oriented Dual Income Tax, July 2005

1514 Fahad Khalil, David Martimort and Bruno Parigi, Monitoring a Common Agent: Implications for Financial Contracting, August 2005

1515 Volker Grossmann and Panu Poutvaara, Pareto-Improving Bequest Taxation, August 2005

1516 Lars P. Feld and Emmanuelle Reulier, Strategic Tax Competition in Switzerland: Evidence from a Panel of the Swiss Cantons, August 2005 
1517 Kira Boerner and Silke Uebelmesser, Migration and the Welfare State: The Economic Power of the Non-Voter?, August 2005

1518 Gabriela Schütz, Heinrich W. Ursprung and Ludger Wößmann, Education Policy and Equality of Opportunity, August 2005

1519 David S. Evans and Michael A. Salinger, Curing Sinus Headaches and Tying Law: An Empirical Analysis of Bundling Decongestants and Pain Relievers, August 2005

1520 Michel Beine, Paul De Grauwe and Marianna Grimaldi, The Impact of FX Central Bank Intervention in a Noise Trading Framework, August 2005

1521 Volker Meier and Matthias Wrede, Pension, Fertility, and Education, August 2005

1522 Saku Aura and Thomas Davidoff, Optimal Commodity Taxation when Land and Structures must be Taxed at the Same Rate, August 2005

1523 Andreas Haufler and Søren Bo Nielsen, Merger Policy to Promote 'Global Players'? A Simple Model, August 2005

1524 Frederick van der Ploeg, The Making of Cultural Policy: A European Perspective, August 2005

1525 Alexander Kemnitz, Can Immigrant Employment Alleviate the Demographic Burden? The Role of Union Centralization, August 2005

1526 Baoline Chen and Peter A. Zadrozny, Estimated U.S. Manufacturing Production Capital and Technology Based on an Estimated Dynamic Economic Model, August 2005

1527 Marcel Gérard, Multijurisdictional Firms and Governments' Strategies under Alternative Tax Designs, August 2005

1528 Joerg Breitscheidel and Hans Gersbach, Self-Financing Environmental Mechanisms, August 2005

1529 Giorgio Fazio, Ronald MacDonald and Jacques Mélitz, Trade Costs, Trade Balances and Current Accounts: An Application of Gravity to Multilateral Trade, August 2005

1530 Thomas Christiaans, Thomas Eichner and Ruediger Pethig, A Micro-Level 'Consumer Approach' to Species Population Dynamics, August 2005

1531 Samuel Hanson, M. Hashem Pesaran and Til Schuermann, Firm Heterogeneity and Credit Risk Diversification, August 2005

1532 Mark Mink and Jakob de Haan, Has the Stability and Growth Pact Impeded Political Budget Cycles in the European Union?, September 2005

1533 Roberta Colavecchio, Declan Curran and Michael Funke, Drifting Together or Falling Apart? The Empirics of Regional Economic Growth in Post-Unification Germany, September 2005 
1534 Kai A. Konrad and Stergios Skaperdas, Succession Rules and Leadership Rents, September 2005

1535 Robert Dur and Amihai Glazer, The Desire for Impact, September 2005

1536 Wolfgang Buchholz and Wolfgang Peters, Justifying the Lindahl Solution as an Outcome of Fair Cooperation, September 2005

1537 Pieter A. Gautier, Coen N. Teulings and Aico van Vuuren, On-the-Job Search and Sorting, September 2005

1538 Leif Danziger, Output Effects of Inflation with Fixed Price- and Quantity-Adjustment Costs, September 2005

1539 Gerhard Glomm, Juergen Jung, Changmin Lee and Chung Tran, Public Pensions and Capital Accumulation: The Case of Brazil, September 2005

1540 Yvonne Adema, Lex Meijdam and Harrie A. A. Verbon, The International Spillover Effects of Pension Reform, September 2005

1541 Richard Disney, Household Saving Rates and the Design of Social Security Programmes: Evidence from a Country Panel, September 2005

1542 David Dorn and Alfonso Sousa-Poza, Early Retirement: Free Choice or Forced Decision?, September 2005

1543 Clara Graziano and Annalisa Luporini, Ownership Concentration, Monitoring and Optimal Board Structure, September 2005

1544 Panu Poutvaara, Social Security Incentives, Human Capital Investment and Mobility of Labor, September 2005

1545 Kjell Erik Lommerud, Frode Meland and Odd Rune Straume, Can Deunionization Lead to International Outsourcing?, September 2005

1546 Robert Inklaar, Richard Jong-A-Pin and Jakob de Haan, Trade and Business Cycle Synchronization in OECD Countries: A Re-examination, September 2005

1547 Randall K. Filer and Marjorie Honig, Endogenous Pensions and Retirement Behavior, September 2005

1548 M. Hashem Pesaran, Til Schuermann and Bjoern-Jakob Treutler, Global Business Cycles and Credit Risk, September 2005

1549 Ruediger Pethig, Nonlinear Production, Abatement, Pollution and Materials Balance Reconsidered, September 2005 